\title{
Association between anterior talofibular ligament injury and ankle tendon, ligament, and joint conditions revealed by magnetic resonance imaging
}

\author{
Israel Casado-Hernández ${ }^{1}{ }^{\wedge}$, Ricardo Becerro-de-Bengoa-Vallejo ${ }^{2} \wedge$, Marta Elena Losa-Iglesias ${ }^{1}$, \\ Fernando Santiago-Nuño ${ }^{3} \wedge$, Victoria Mazoteras-Pardo ${ }^{2} \wedge$, Daniel López-López $^{3} \wedge$, David Rodríguez-Sanz ${ }^{2}$, \\ César Calvo-Lobo ${ }^{2} \wedge$ \\ ${ }^{1}$ Faculty of Health Sciences, Universidad Rey Juan Carlos, Spain; ${ }^{2}$ Facultad de Enfermería, Fisioterapia y Podología. Universidad Complutense de \\ Madrid, Spain; ${ }^{3}$ Research, Health and Podiatry Group, Department of Health Sciences, Faculty of Nursing and Podiatry, Universidade da Coruña, \\ Ferrol, Spain
}

Correspondence to: Dr. David Rodríguez-Sanz, PhD. Facultad de Enfermería, Fisioterapia y Podología. Universidad Complutense de Madrid, Spain, Plaza de Ramón y Cajal, 3, Madrid, Spain. Email: davidrodriguezsanz@ucm.es.

\begin{abstract}
Background: The lateral ankle ligament complex is the most frequently injured ligament secondary to strong ankle inversion movement during lateral ankle sprains (LAS). Among these injuries, anterior talofibular ligament (ATFL) injury is the most frequent condition (present in 66-85\% of such injuries). The purpose of this research was to use magnetic resonance imaging (MRI) to determine the association between ankle tendon, ligament, and joint conditions and ATFL injuries.

Methods: A case-control MRI study was carried out to compare the presence of ankle muscle, tendon, ligament, and joint conditions in patients with injured ATFLs (case group; $n=25$ ) and non-injured ATFLs (control group; $\mathrm{n}=25$ ).
\end{abstract}

Results: Achilles tendinopathy was present in 1/25 (4\%) patients with injured ATFLs and 7/25 (28\%) noninjured ATFL subjects ( $\mathrm{P}=0.048)$. Injured calcaneofibular ligaments (CFLs) were present in 19/25 (76\%) patients with injured ATFLs and 1/25 (4\%) non-injured ATFL subjects $(\mathrm{P}<0.001)$. Finally, injured tibiotalar joints were present in 16/25 (64\%) patients with injured ATFLs and 5/25 (20\%) non-injured ATFL subjects $(\mathrm{P}=0.002)$. Other musculoskeletal structure injuries occurred at similar rates between patients with injured ATFLs and those with non-injured ATLFs $(\mathrm{P} \geq 0.05)$.

Conclusions: Patients with ATFL injuries showed a greater presence of CFL and tibiotalar joint injuries than subjects with non-injured ATFLs.

Keywords: Ankle injuries; magnetic resonance imaging (MRI); musculoskeletal diseases; sprains and strains

Submitted Jan 02, 2020. Accepted for publication Jul 08, 2020.

doi: 10.21037/qims-20-5

View this article at: http://dx.doi.org/10.21037/qims-20-5

\footnotetext{
^ ORCID: Israel Casado-Hernández, 0000-0002-8537-425X; Ricardo Becerro-de-Bengoa-Vallejo, 0000-0003-1568-7602; Marta Elena Losa-Iglesias, 0000-0001-7588-2069; Fernando Santiago-Nuño, 0000-0001-5597-8063; Victoria Mazoteras-Pardo, 0000-0001-5741-7606; Daniel López-López, 0000-0002-9818-6290; César Calvo-Lobo, 0000-0002-6569-1311.
} 


\section{Introduction}

The lateral ankle ligament complex is comprised of the anterior talofibular ligament (ATFL), calcaneofibular ligament (CFL), and posterior talofibular ligament (PTFL) (1). The key function of the lateral ankle ligament complex is the avoidance of sudden ankle inversion movements (2). Lateral ankle ligament complex injuries are present in up to $85 \%$ of ankle sprains, making it the most frequently injured ligament secondary to strong ankle inversion movement during lateral ankle sprains (LAS) (3). Under abrupt inversion mechanisms, the CFL may be injured in conjunction with the ATFL, reaching a prevalence of $20 \%$ to $40 \%$ (1), whereas PTFL injury is infrequent (2), being the ATFL considered as the principal injured ligament followed by the CFL $(4,5)$. From $20 \%$ to $40 \%$ about subjects with acute LAS usually developed chronic ankle instability (6-8).

Leardini et al. (9) carried out a cadaveric study in order to predict the function of each lateral ankle complex ligament under different flexion angles using the functional length fraction obtained by the relationship between the longitudinal ligament mean and the maximum longitudinal elongation. These authors concluded that the CFL played a key role in flexion and extension ankle stabilization.

In our study, the medial ankle complex is comprised of the tibialis posterior tendon, spring ligament (SL), deltoid ligament and tibiotalar ligament (10). Patients with a progressive tibialis posterior degeneration and dysfunction are related with a frequently damaged SL developing ankle instability $(11,12)$

Indeed, the SL may be considered a passive stabilizer of the plantar arch in the talocalcaneal joint that helps to avoid talus medial rotation and plantar flexion, as well as navicular dorsal flexion, eversion, and abduction (13). In one study, SL injury seemed to be produced in the upper-medial bundles due to its anatomic disposition, hammock shape, and lack of vascularization. The SL may be considered the strongest medial ligament since this ligament supports compression forces (generated between the tibialis posterior tendon and talus head) and elongation forces (14). Regarding conditions secondary to SL injuries, adult acquired pes planus may be produced by isolated SL injuries (15-19). Also, the posterior tibialis tendon may be considered an active stabilizer of the plantar arch and its dysfunction may commonly lead to secondary injury of the SL and talocalcaneal ligaments; it may also be linked to injuries of the deltoid ligament, the plantar fascia, and other musculoskeletal soft-tissue structures (20).

From a biomechanical point of view, functional alterations of the ATFL, CFL, and talocalcaneal interosseous ligament may provoke a non-physiological talus anterolateral rotation during gait cycle secondary to talocalcaneal joint instability (21-23). Magnetic resonance imaging (MRI) is a precise, reliable, and valid tool to visualize and diagnose ankle injuries (24). Nevertheless, there is a lack of MRI-based research studies that link the presence of ATFL injuries to other musculoskeletal injuries.

We hypothesized ATFL injury produced ankle instability and develop ligament, tendon and joint injuries of the lateral and medial ankle complex.

Therefore, the purpose of this study was to determine if there are any associations between ankle tendon, ligament, and joint conditions and ATFL injuries that are revealed by MRI.

\section{Methods}

\section{Design and sample}

A case-control study was carried out in order to compare, via MRI, the presence of ankle tendon, ligament, and joint conditions in injured and non-injured ATFLs according to the Strengthening the Reporting of Observational studies in Epidemiology (STROBE) guidelines (25). Researchers accessed medical records and a MRI database from a podiatric outpatient clinic, using the terms "ATFL, "sprains" and "rupture", and found 50 MRIs and medical records, 25 with injured ATFLs and 25 non-injured ATFLs, that included data collected by a consecutive sampling method. Thus, the inclusion criteria were an indication of ankle MRI included in the MRI database of the podiatric outpatient clinic using the described search terms ("ATFL", "sprains", and "rupture"). The exclusion criteria were the presence of fractures, systemic conditions, rheumatic or autoimmune diseases, and/or neuropathies in the ankle, as well as other musculoskeletal conditions located in the lower limbs that did not involve the ankle, in the medical record.

\section{Ethical considerations}

This study was approved by the Ethics Committee of Rey Juan Carlos University (Spain; Registration number of 0801201800618). The Helsinki declaration and the ethical recommendations for biomedical research were respected (26). The patients previously provided informed consent at the 


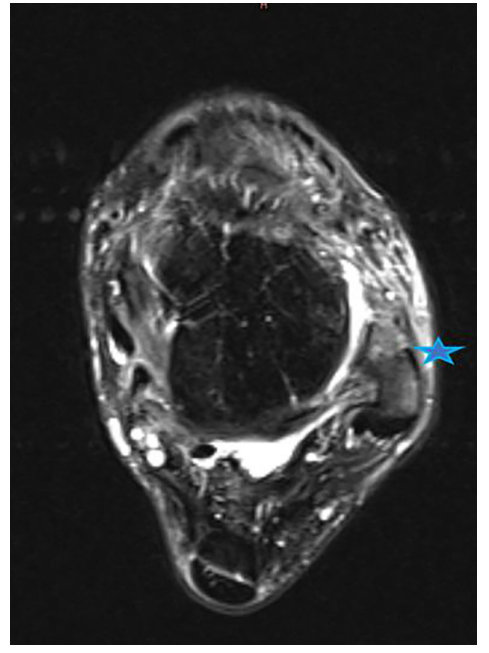

Figure 1 Axial fat-sat proton-density image. Blue star shows anterior talofibular ligament complete rupture in distal fibula portion with subcutaneous cell tissue edema.

podiatric outpatient clinic.

\section{Procedure}

Musculoskeletal structures were evaluated by MRI, such as tendons (flexor hallucis and digitorum tendons, extensor hallucis and digitorum tendons, tibialis anterior tendon, Achilles tendon and plantar fascia, and peroneus longus and brevis tendons), ligaments (PTFL, CFL, ATFL, SL, and deltoid ligament), and joints (talocalcaneal joint and tibiotalar joint).

Tendinosis was defined as the tendon intrasubstance signal intensity increment in longitudinal relaxed time (T1) and proton density (DP) and increased thickness in the axial and coronal planes without an increase in peritendinous edema (27). "Partial injury" was defined as more evident morphologic and signal intensity alterations that were associated with heterogeneous linear images that did not show a complete tear (28). In addition, alterations in the sequence T2 signal intensity were interpreted as "partial injuries" or advanced intrasubstance degeneration (tendinosis) (29). "Partial rupture" was defined as a focal increase in the $\mathrm{DP}$ and T1 sequence signal intensity (and occasionally T2 sequence intensity) $(27,29,30)$. "Complete rupture" was defined as the total disruption of tendon fibers (29). Joints injuries, such as talocalcaneal and tibiotalar lesions, were determined as joint rupture, osteochondral injury, osteonecrosis and/or synovitis. Peritendinous edema quantity was not considered due to the high variability of this variable in prior research studies, including in healthy subjects $(28,31,32)$. Notably, non-significant issues associated with a clinical indicator (cutaneous marker) coincided with a slight increase in peritendinous edema, without signal intensity changes or morphological changes in the adjacent tendon. If the edema surrounded the tendon completely, except an isolated defined sector ("tendon mesentery"), these changes were classified as tenosynovitis (33). In this study, data compatible with prior muscle, joint, or ligament ruptures were not considered. Fasciosis was defined as the focal or continuous increase in the thickness $(>4 \mathrm{~mm}$ ) of bundles in its proximal insertion (34). Achilles tendinopathy was defined as tendon thickening $(>8 \mathrm{~mm}$ in the anteroposterior plane), with or without intrasubstance signal alterations, peritendinosis, or partial or complete rupture (34).

Synovitis was defined as synovial membrane thickening in T1-boosted sequences and increased signals in T2boosted sequences (35). Representative MRI imagines are presented in Figures 1-10 in order to illustrate the definition of each condition.

\section{MRI processing}

MRI data were analyzed by a 1.5 -T scanner measurement system (Signa HD 1.5T RM, GE Medical Systems, Milwaukee, WI, USA). Images were separately analyzed by two radiologists who specialized in musculoskeletal analyses with more than 10 years of experience. Both radiologists were blinded to the case or control group allocation when analyzing the MRI data.

All MRI measurements were carried out under the following sequences in the axial, sagittal, and coronal planes: 1.5-T MRI (General Electric Medical Systems, Milwaukee, WI), T1-weighted spin echo [400-600 ms/9-17 ms (TR/ TE range); $256 \times 192$ matrix; from 12- to $16-\mathrm{cm}$ FOV; 1-2 NEX, 3-mm thickness cut; 1-mm space], T2-weighted spin echo [2,000-3,000 ms/70-80 ms (TR/TE range); 256×192 matrix from 12- to 16-cm FOV; 1 NEX, 3-mm thickness; 1-mm space], T2-weighted spin echo with fat saturation [3,083-5,000 ms/80-108 ms (TR/TE range); 256×192 matrix from 12- to 16-cm FOV; 2-3 NEX, 3-mm thickness; 1-mm space] and DP fast spin echo with fat saturation [1,800-3,050 ms/15-40 ms (TR/TE range)]; 256×192 matrix; 12-16 FOV; 1 NEX; 3-mm thickness cut and 1-mm space. Not all sequences were carried out in all patients but all the protocols were the same for all patients (36). 


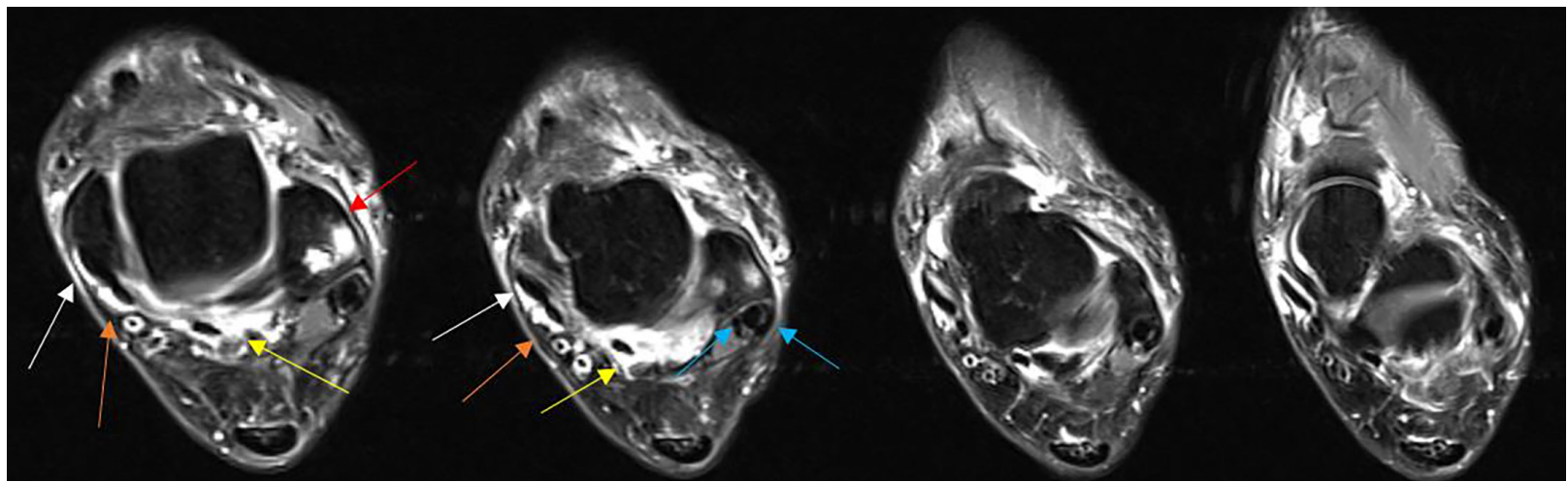

Figure 2 Axial fat-sat proton-density image. Blue arrow shows peroneus brevis tendon partial rupture with a characteristic of "V" shape surrounding a thickened peroneus longus tendon. Red arrow shows fibularis head cyst lesion. Yellow arrow shows hallucis longus tendon partial injury (tenosynovitis). Orange arrow shows partial flexor digitorum tendon injury (tenosynovitis) and white arrow show partial posterior tibialis tendon injury (tenosynovitis).
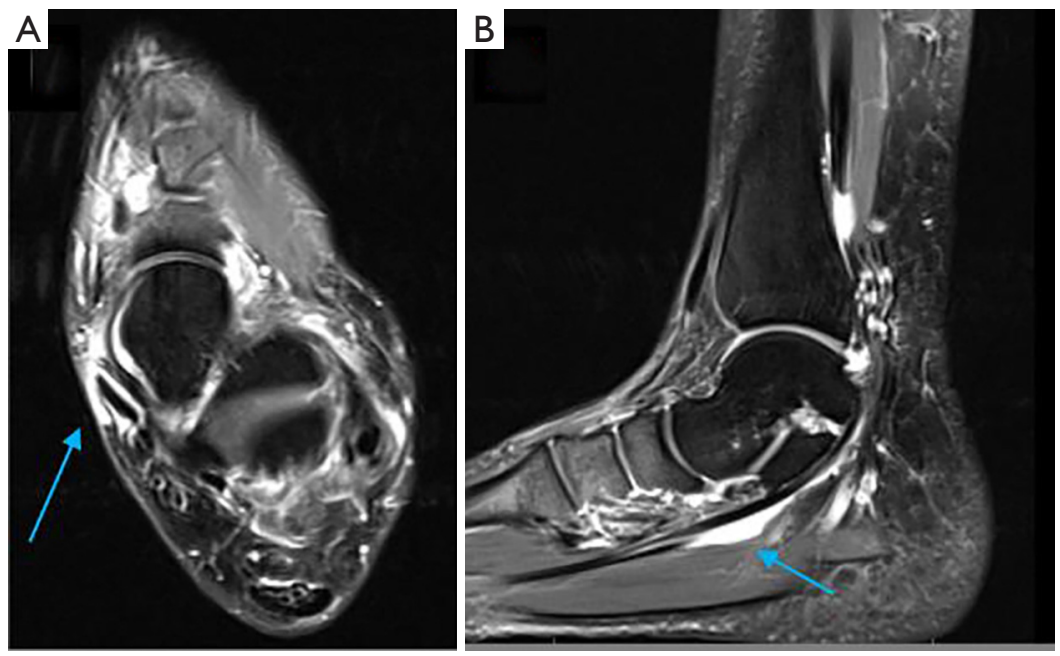

Figure 3 Partial tibialis posterior tendon injury image at the medial part of the ankle. (A) Axial fat-sat proton-density. Blue arrow shows partial tibialis posterior tendon injury (tenosynovitis). (B) Sagittal fat-sat proton-density blue arrow shows partial tibialis posterior tendon injury (tenosynovitis).

\section{Sample size calculation}

The CFL injury proportion was considered the main outcome measurement because this ligament seemed to be commonly injured in conjunction with the ATFL (1); therefore, a chi-squared test for Goodness of Fit was used for the sample size calculation in $G^{*}$ Power in order to test the null hypothesis that the observed frequencies would be equal to the expected frequencies (37). Based on a prior pilot study $(\mathrm{n}=18)$ that evaluated a non-injured ATFL control group (including 1 injured CFL from 9 feet) and an injured ATFL case group (including 3 injured CFLs from 9 feet), the normalized expected proportions for each category were $\mathrm{P}\left(\mathrm{H}_{0}\right)=0.5$ for the case group and $\mathrm{P}\left(\mathrm{H}_{0}\right)=0.5$ for the control group and the normalized observed proportions were $\mathrm{P}\left(\mathrm{H}_{1}\right)=0.25$ for the control group and $\mathrm{P}\left(\mathrm{H}_{1}\right)=0.75$ for the case group. According to an effect size of $W=0.50$, an error probability of $\alpha=0.05$, a power ( $1-\beta$ error probability) of 0.90 , and 1 degree of freedom, there was a $90 \%$ chance of correctly rejecting the null hypothesis of no difference between the expected and observed proportions with 43 participants (critical $\chi^{2}=3.84$ ). 


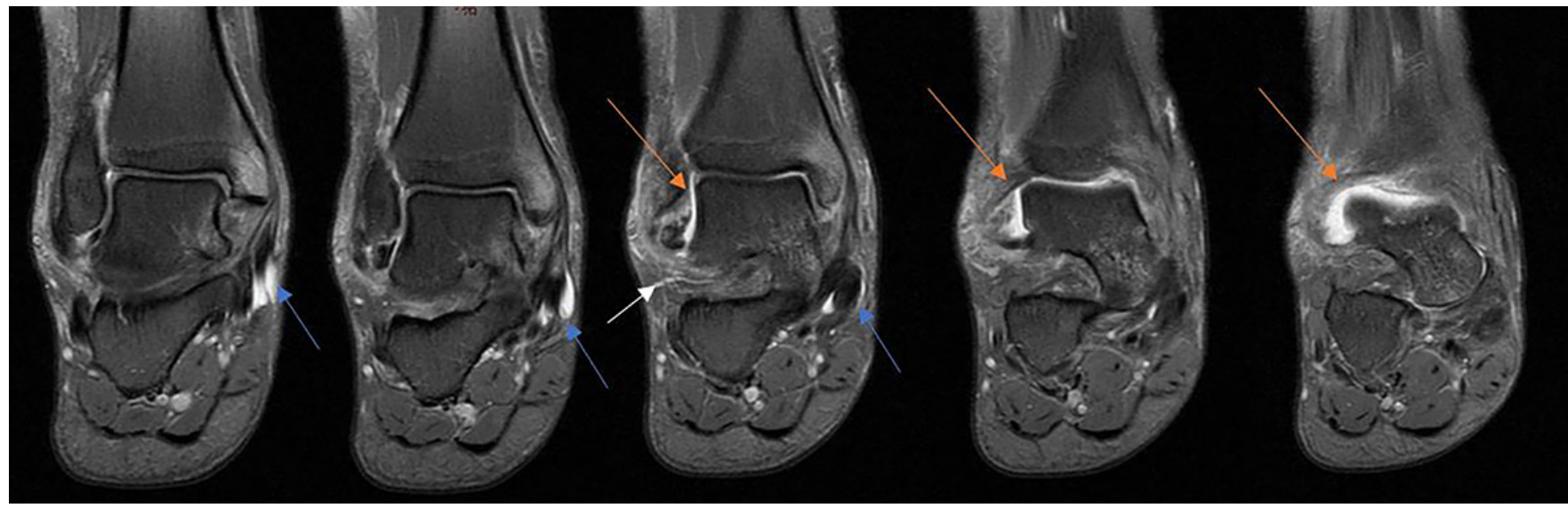

Figure 4 Axial fat-sat proton-density. Orange arrows show partial tibiotalar joint injury (synovitis). White arrow shows partial calcaneofibular injury. Blue arrows show partial tibialis posterior tendon injury (tenosynovitis).

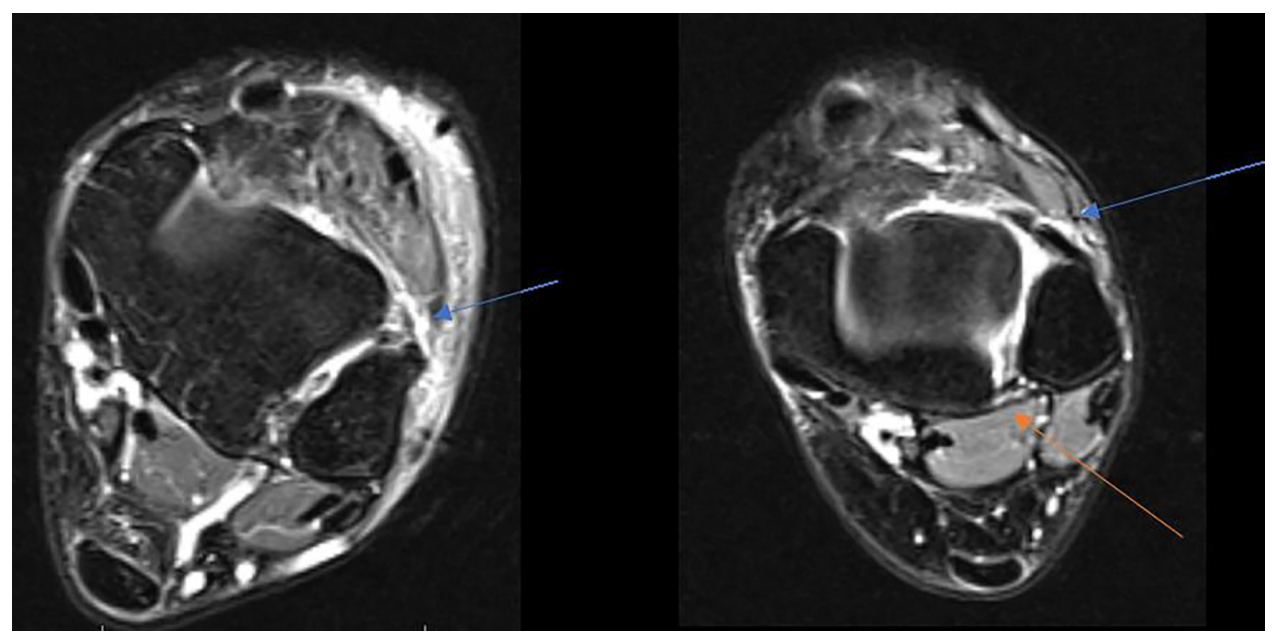

Figure 5 Axial fat-sat proton-density. Blue arrows show complete anterior talofibular ligament rupture. Orange arrow shows partial posterior talofibular ligament injury.

\section{Statistical analysis}

Statistical analysis was performed using the SPSS 19.0 statistical software for Windows (SPSS Inc., Chicago, USA). First, the Shapiro-Wilk test was used to test if the data were normally distributed. All the variable values were considered parametric data since a normal distribution was shown (according to a $\mathrm{P}$ value $>0.05$ in the Shapiro-Wilk test). Second, mean \pm standard deviation (SD) and the upper and lower limits for the $95 \%$ confidence interval (CI) were utilized in order to describe the quantitative data. Third, differences between the case and control groups were compared by Student's $t$-test for independent samples for parametric data. Finally, the categorical data were described as frequencies and percentages (\%) and their differences between the case and control groups were compared using Fisher's exact test for dichotomous variables. Statistical significance was set at a $\mathrm{P}$ value $<0.05$ for a $95 \%$ CI.

\section{Results}

\section{Sociodemographic data}

The case group's sociodemographic data showed a mean age of $41.44 \pm 14.89$ years (95\% CI: 35.29 to 47.59 years), a mean height of $171.64 \pm 5.61 \mathrm{~cm}(95 \% \mathrm{CI}: 169.32$ to $173.96 \mathrm{~cm})$, 


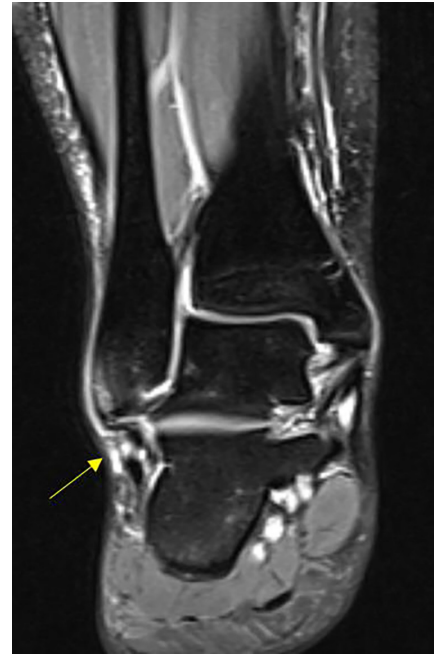

Figure 6 Coronal fat-sat proton-density. Yellow arrow shows partial fibulocalcaneal tendon injury.

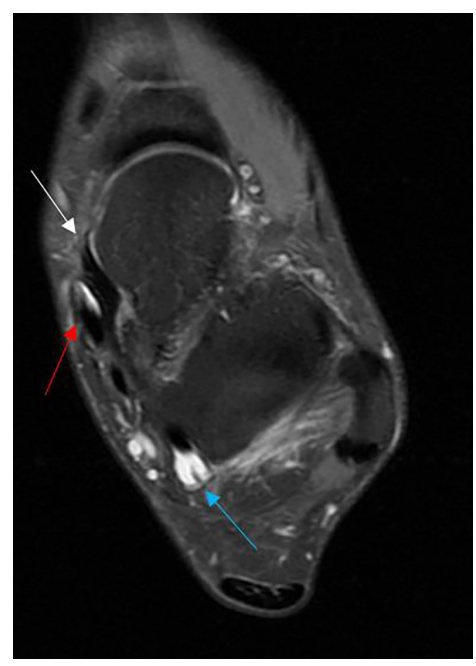

Figure 7 Axial fat-sat proton-density. White arrow shows spring ligament injury (thickened). Blue arrow shows flexor hallucis longus tendon injury (tenosynovitis). Red arrow shows tibialis posterior tendon injury (tenosynovitis).

a mean weight of $66.64 \pm 10.11 \mathrm{~kg}$ (95\% CI: 62.47 to $70.81 \mathrm{~kg})$, and a mean body mass index (BMI) of $22.71 \pm 2.91 \mathrm{~kg} / \mathrm{cm}^{2}$ (95\% CI: 21.50 to $23.91 \mathrm{~kg} / \mathrm{cm}^{2}$ ). The control group's sociodemographic data showed a mean age of $45.68 \pm 11.96$ years (95\% CI: 40.74 to 50.62 years), a mean height of $170.96 \pm 6.41 \mathrm{~cm}$ (95\% CI: 168.31 to $173.61 \mathrm{~cm}$ ), a mean weight of 69.64 \pm 8.55 (95\% CI: 66.11 to $73.17 \mathrm{~kg}$ ), and a mean BMI of $24.12 \pm 2.54 \mathrm{~kg} / \mathrm{cm}^{2}$ (95\% CI: 23.07 to $25.17 \mathrm{~kg} / \mathrm{cm}^{2}$ ).

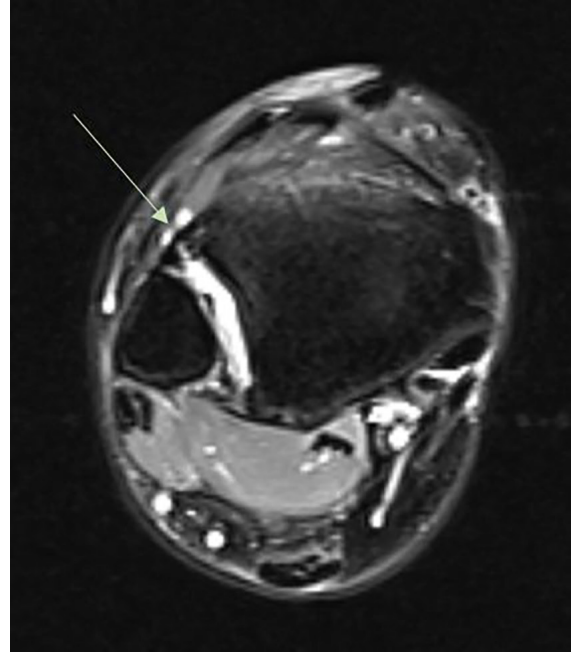

Figure 8 Axial fat-sat proton-density. Green arrow shows partial anterior tibiofibular ligament rupture with edema.

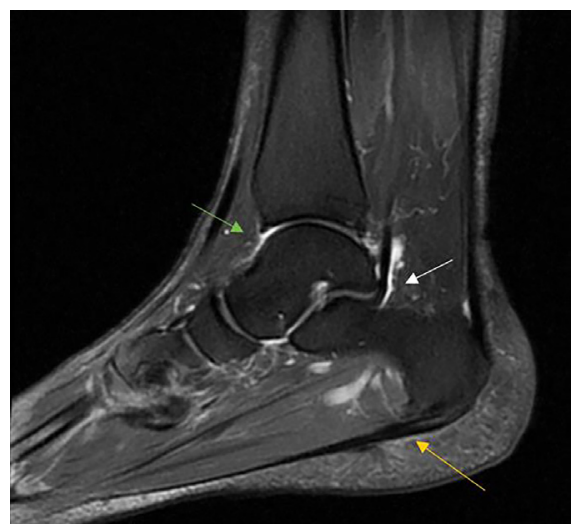

Figure 9 Sagittal fat-sat proton-density. Green arrow shows tibiotalar synovitis. White arrow shows tenosynovitis of the tibialis posterior tendon. Orange arrow shows a thickened plantar fascia.

In addition, there were 24 (48\%) women and $26(52 \%)$ men in the total sample, 12 (48\%) women and $13(52 \%)$ men in each group. The age, height, weight, and BMI differences between the groups were not statistically significant (Table 1).

\section{MRI findings}

In the case group, which was composed of 25 patients with ATFL injuries, the MRI findings showed 4 (16\%) grade-I sprains, 3 (12\%) grade-II sprains, 1 (4\%) grade-III sprain, $11(44 \%)$ chronic sprains, and $6(24 \%)$ complete ruptures.

Table 2 shows the frequencies and percentages of other 
musculoskeletal injuries in patients with injured ATFLs (case group) and healthy subjects without ATFL injuries (control group). Statistically significant differences were found for the presence of Achilles tendinopathy [1/25 (4\%) patients with injured ATFLs and 7/25 (28\%) non-injured ATFL subjects; $\mathrm{P}=0.048]$, injured CFLs [19/25 (76\%) patients with injured ATFLs and 1/25 (4\%) non-injured ATFL subjects; $\mathrm{P}<0.001]$, and injured tibiotalar joints $[16 / 25$ (64\%) patients with injured ATFLs and 5/25 (20\%) non-injured ATFL subjects; $\mathrm{P}=0.002]$.

Other musculoskeletal structures, such as the flexor hallucis longus tendon, flexor digitorum longus tendon, extensor hallucis longus tendon, extensor digitorum longus tendon, tibialis posterior tendon, tibialis anterior tendon, peroneus longus and brevis tendons, plantar fascia, PTFL, anterior tibiofibular ligament, tibiotalar ligament, SL, deltoid ligament, and talocalcaneal joint did not show statistically significant differences $(\mathrm{P}>0.05)$ between the two groups.

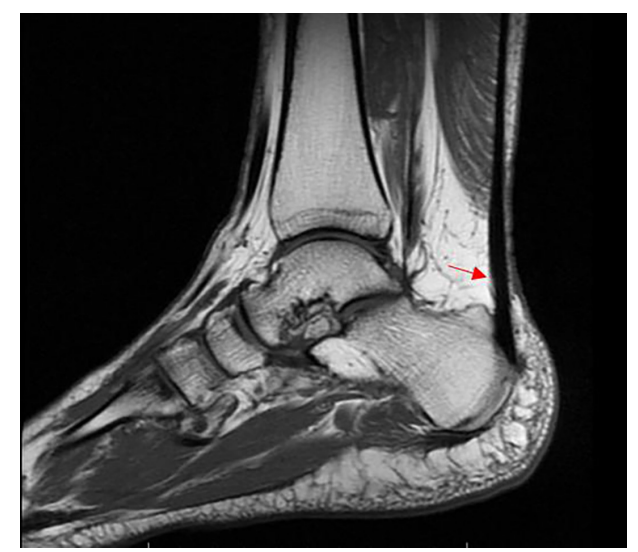

Figure 10 Sagittal fast spin echo T1. Red arrow shows a thickened Achilles tendon.

\section{Discussion}

To the authors' knowledge, this study showed that patients who suffered from ATFL injuries exhibited a greater frequency of CFL and tibiotalar joint injuries than subjects who did not suffer from ATFL injuries. A recent study about the epidemiology of injuries secondary to ankle sprains diagnosed in an orthopedic emergency service concluded that approximately $92 \%$ of their patients suffered from some kind of injury according to their MRI findings, as indicated by correlations between lateral ligament complex injuries and syndesmosis and deltoid injuries, but not between deltoid and syndesmosis conditions, lateral ligamentous and talocalcaneal injuries, or ankle and midfoot injuries (38). Even though higher incidences of CFL and tibiotalar injuries were previously reported in patients with ATFL injuries, our study may be the first case-control study to focus solely on ATFL injury and its relationship with several tendon, ligament, and joint structures. Indeed, among the parts of the ankle lateral ligamentous complex, the ATFL is considered the ligament structure that is most frequently injured during sudden inversion movements of the ankle (1), justifying our investigation.

\section{Association between tendon, ligament, and joint conditions and ATFL injury}

Firstly, our findings about the positive association between CFL and ATFL injuries as the main outcome measurements are supported by previous studies that reported that the CFL is commonly injured in conjunction with the ATFL under abrupt inversion mechanisms (1). Secondly, our findings regarding the positive association between tibiotalar joint and ATFL injuries may be in accordance with prior studies that reported subchondral and "kissing"

Table 1 Demographic data of the total sample, case and control groups

\begin{tabular}{lcccc}
\hline & Total sample $(\mathrm{n}=50)$, mean \pm SD; & Cases group & & Control group \\
\cline { 3 - 4 } Demographic data & $(95 \% \mathrm{Cl})$ & $\begin{array}{c}\text { Injured ATFL }(\mathrm{n}=25), \\
\text { mean } \pm \text { SD; }(95 \% \mathrm{Cl})\end{array}$ & $\begin{array}{c}\text { Non-injured ATFL }(\mathrm{n}=25), \\
\text { mean } \pm \text { SD; (95\% Cl) }\end{array}$ \\
\hline Age (years) & $43.52 \pm 13.61 ;(39.65-47.39)$ & $41.44 \pm 14.89 ;(35.29-47.59)$ & $45.68 \pm 11.96 ;(40.74-50.62)$ & 0.266 \\
Height $(\mathrm{cm})$ & $171.30 \pm 5.97 ;(169.60-173.00)$ & $171.64 \pm 5.61 ;(169.32-173.96)$ & $170.96 \pm 6.41 ;(168.31-173.61)$ & 0.263 \\
Weight $(\mathrm{kg})$ & $68.14 \pm 9.39 ;(65.47-70.81)$ & $66.64 \pm 10.11 ;(62.47-70.81)$ & $69.64 \pm 8.55 ;(66.11-73.17)$ & 0.692 \\
BMI $\left(\mathrm{kg} / \mathrm{m}^{2}\right)$ & $23.42 \pm 2.80 ;(22.62-24.21)$ & $22.71 \pm 2.91 ;(21.50-23.91)$ & $24.12 \pm 2.54 ;(23.07-25.17)$ & 0.073 \\
\hline
\end{tabular}

*, student $t$ test for independent samples was used. Statistically significance was set at $\mathrm{P}$ value $<0.05$ for a $95 \% \mathrm{Cl}$. ATFL, anterior talofibular ligament; BMI, body mass index; $\mathrm{Cl}$, confidence interval. 
Table 2 Comparisons of injured and non-injured musculoskeletal structures with and without ATFL injury

\begin{tabular}{|c|c|c|c|}
\hline Musculoskeletal structures & Control group (non-injured ATFL) [\%] & Case group (injured ATFL) [\%] & $P$ value* \\
\hline Injured flexor hallucis longus tendon & $6[24]$ & $1[4]$ & 0.179 \\
\hline Non-injured flexor hallucis longus tendon & $19[76]$ & 24 [96] & \\
\hline Injured flexor digitorum longus tendon & $3[12]$ & $3[12]$ & 1 \\
\hline Non-injured flexor digitorum longus tendon & $22[88]$ & 22 [88] & \\
\hline Injured extensor hallucis longus tendon & $0[0]$ & $0[0]$ & 1 \\
\hline Non-injured extensor hallucis longus tendon & 25 [100] & $25[100]$ & \\
\hline Injured tibialis posterior tendon & $7[28]$ & $5[20]$ & 0.741 \\
\hline Injured plantar fascia & $5[20]$ & $3[12]$ & 0.701 \\
\hline Non-injured plantar fascia & $20[80]$ & $22[88]$ & \\
\hline Injured Achilles tendon & $7[28]$ & $1[4]$ & 0.048 \\
\hline Non-injured Achilles tendon & $18[72]$ & $24[96]$ & \\
\hline Injured peroneus brevis tendon & $4[16]$ & $5[20]$ & 1 \\
\hline Non-injured peroneus brevis tendon & $21[84]$ & $20[80]$ & \\
\hline Injured peroneus longus tendon & $0[0]$ & $5[20]$ & 0.05 \\
\hline Non-injured peroneus longus tendon & $25[100]$ & $20[80]$ & \\
\hline Non-injured anterior tibiofibular ligament & $25[100]$ & 23 [92] & \\
\hline Injured tibiotalar ligament & $4[16]$ & $7[28]$ & 0.496 \\
\hline Non-injured tibiotalar ligament & $21[84]$ & $18[72]$ & \\
\hline Injured SL & $6[24]$ & $12[48]$ & 0.254 \\
\hline Non-injured SL & $19[76]$ & $13[52]$ & \\
\hline Injured deltoid ligament & $2[8]$ & $2[8]$ & 1 \\
\hline Non-injured deltoid ligament & 23 [92] & 23 [92] & \\
\hline Injured tibiotalar joint & $5[20]$ & 16 [64] & 0.002 \\
\hline Non-injured tibiotalar joint & $20[80]$ & $9[36]$ & \\
\hline Injured talocalcaneal joint & $4[16]$ & $9[36]$ & 0.196 \\
\hline Non-injured talocalcaneal joint & $21[84]$ & 16 [64] & \\
\hline
\end{tabular}

*, Fisher exact test was used. Statistically significance was set at $\mathrm{P}$ value $<0.05$ for a $95 \% \mathrm{Cl}$. ATFL, anterior talofibular ligament; Cl, confidence interval; CFL, calcaneofibular ligament; PTFL, posterior talofibular ligament; SL, Spring ligament. 
lesions in the talus and tibia after ankle trauma in $18 \%$ and $50 \%$ of acute and recurrent sprains, respectively (39). Finally, our last finding was a negative association between Achilles tendon and ATFL injuries since Achilles tendon injuries were more common in the control group, possibly because our sample control group presented higher average age, weight, height, and BMI values, although without statistically significant differences, than our case group and some of the reported demographic data were previously identified as risk factors for Achilles tendon abnormalities $(40,41)$. There is a lack of studies reported association between ATFL injuries and Achilles tendon injuries. Dreyer et al. referred an uncommon and isolated Achilles rupture with associated with clinical symptoms of LAS (42). Lam et al. reported a case study of undiagnosed rupture of the Achilles tendon related to severe LAS (43).

\section{Non-association between tendon, ligament, and joint conditions and ATFL injury}

Other musculoskeletal structures, such as some tendons (flexor hallucis longus, flexor digitorum longus, extensor hallucis longus, extensor digitorum longus, tibialis posterior, tibialis anterior, peroneus longus and brevis tendons, and plantar fascia), ligaments (PTFL, anterior tibiofibular ligament, tibiotalar ligament, SL, and deltoid ligament), and the talocalcaneal joint, did not show any relationship with ATLF injuries. Our findings regarding these musculoskeletal structures are in line with prior research $(2,38)$, except that a previous study observed correlations between lateral ligament complex injuries and syndesmosis and deltoid injuries (38) and we did not. A possible explanation of these controversial findings may be that our study was carried out in a podiatric outpatient clinic, whereas the other study was performed in an orthopedic emergency room (38). Ziai et al. analyzed the influence of the peroneus longus as passive ankle stabilizer in lateral sprains being closing that the peroneus longus tendon assume the role of stabilizer in ankle sprains (44). In our study, the peroneus longus tendon has statistically significance only in the $20 \%$ ATLF injuries. More research is necessary in this way. Recent research of Abdeen et al. compared ankle structures injured and non-injured in LAS, concluded no statistically significant differences between health injured tendon and muscles ankle with the injured, these results are similar as our findings (45).

\section{Clinical implications}

Our study's findings support that the idea CFL and tibiotalar joint injuries should be systematically explored via ankle MRI in patients who suffer from ATLF injuries due to their presented association. Regarding the pathophysiology of the ATFL, ATLF injury seemed to be linked to ankle inversion mechanisms that impair the CFL and tibiotalar joint structures, which partially explains why other musculoskeletal structures, such as the deltoid ligament, and anterior tibiofibular injuries did not show a clear relationship with ATLF injury (38).

\section{Limitations}

There were some limitations to this study that should be acknowledged. First, a consecutive sampling method was used to select the participants and future studies should use randomized sampling methods. Second, the present study only permits us to state associations and future cohort studies should be designed in order to determine causal relationships. Third, despite the lack of statistically significant demographic differences between the case and control groups, our between-group differences could be under-estimated and influenced by the control group's higher age and BMI, compared to the case group, which are known risk factors associated with a higher prevalence of MRI injury findings in the ankle $(46,47)$. Finally, several patients who suffer from ATLF injuries do not receive a medical indication for MRI or other indications that prompt MRI, such as persistent pain or instability; MRI should be considered for these groups in future research studies. Though we performed a sample size calculation, both groups were heterogeneous; the ATFL case group included patients with any grade or chronicity of ATFL injury and the nonATFL injury control group included a heterogeneous mix of other causes of ankle conditions. Therefore, a greater sample size should be considered for future studies.

\section{Conclusions}

Patients who suffered from ATFL injuries showed a greater presence of CFL and tibiotalar joint injuries, compared to subjects who did not suffer from an ATFL injury. Linking evidence to action, these specific musculoskeletal structures should be accurately assessed via MRI study of patients with ATLF injuries.

\section{Acknowledgments}

Funding: None. 


\section{Footnote}

Conflicts of Interest: All authors have completed the ICMJE uniform disclosure form (available at http://dx.doi. org/10.21037/qims-20-5). The authors have no conflicts of interest to declare.

Ethical Statement: This study was approved by the Ethics Committee of Rey Juan Carlos University (Spain; Registration number of 0801201800618 ). The patients previously provided informed consent at the podiatric outpatient clinic.

Open Access Statement: This is an Open Access article distributed in accordance with the Creative Commons Attribution-NonCommercial-NoDerivs 4.0 International License (CC BY-NC-ND 4.0), which permits the noncommercial replication and distribution of the article with the strict proviso that no changes or edits are made and the original work is properly cited (including links to both the formal publication through the relevant DOI and the license). See: https://creativecommons.org/licenses/by-nc-nd/4.0/.

\section{References}

1. Broström L. Sprained ankles. V. Treatment and prognosis in recent ligament ruptures. Acta Chir Scand 1966;132:537-50.

2. Broström L. Sprained ankles. VI. Surgical treatment of "chronic" ligament ruptures. Acta Chir Scand 1966;132:551-65.

3. Garrick JG, Requa RK. The epidemiology of foot and ankle injuries in sports. Clin Sports Med 1988;7:29-36.

4. Kumai T, Takakura Y, Rufai A, Milz S, Benjamin M. The functional anatomy of the human anterior talofibular ligament in relation to ankle sprains. J Anat 2002;200:457-65.

5. Hertel J. Functional Anatomy, Pathomechanics, and Pathophysiology of Lateral Ankle Instability. J Athl Train 2002;37:364-75.

6. Jung HG, Kim NR, Kim TH, Eom JS, Lee DO. Magnetic Resonance Imaging and Stress Radiography in Chronic Lateral Ankle Instability. Foot Ankle Int 2017;38:621-6.

7. Al-Mohrej OA, Al-Kenani N. Chronic ankle instability: Current perspectives. Avicenna J Med 2016;6:103.

8. Sammarco VJ. Complications of lateral ankle ligament reconstruction. In: Clinical Orthopaedics and Related Research. Lippincott Williams and Wilkins, 2001:123-32.

9. Leardini A, O'Connor JJ, Catani F, Giannini S. Kinematics of the human ankle complex in passive flexion; a single degree of freedom system. J Biomech 1999;32:111-8.

10. Boss AP, Hintermann B. Anatomical study of the medial ankle ligament complex. Foot Ankle Int 2002;23:547-53.

11. Kelly M, Masqoodi N, Vasconcellos D, Fowler X, Osman WS, Elfar JC, Olles MW, Ketz JP, Flemister AS, Oh I. Spring ligament tear decreases static stability of the ankle joint. Clin Biomech (Bristol, Avon) 2019;61:79-83.

12. Deland JT, De Asla RJ, Sung IH, Ernberg LA, Potter HG. Posterior tibial tendon insufficiency: Which ligaments are involved? Foot Ankle Int 2005;26:427-35.

13. Deland JT, Arnoczky SP, Thompson FM. Adult acquired flatfoot deformity at the talonavicular joint: reconstruction of the spring ligament in an in vitro model. Foot Ankle 1992;13:327-32.

14. Davis WH, Sobel M, DiCarlo EF, Torzilli PA, Deng X, Geppert MJ, Patel MB, Deland J. Gross, histological, and microvascular anatomy and biomechanical testing of the spring ligament complex. Foot ankle Int 1996;17:95-102.

15. Borton DC, Saxby TS. Tear of the plantar calcaneonavicular (spring) ligament causing flatfoot. A case report. J Bone Joint Surg Br 1997;79:641-3.

16. Tryfonidis M, Jackson W, Mansour R, Cooke PH, Teh J, Ostlere S, Sharp RJ. Acquired adult flat foot due to isolated plantar calcaneonavicular (spring) ligament insufficiency with a normal tibialis posterior tendon. Foot Ankle Surg 2008;14:89-95.

17. Weerts B, Warmerdam PE, Faber FWM. Isolated spring ligament rupture causing acute flatfoot deformity: case report. Foot ankle Int 2012;33:148-50.

18. Masaragian HJ, Ricchetti HO, Testa C. Acute isolated rupture of the spring ligament: a case report and review of the literature. Foot ankle Int 2013;34:150-4.

19. Orr JD, Nunley JA. Isolated Spring Ligament Failure as a Cause of Adult-Acquired Flatfoot Deformity. Foot Ankle Int 2013;34:818-23.

20. Flores DV, Gómez CM, Hernando MF, Davis MA, Pathria MN. Adult acquired flatfoot deformity: Anatomy, biomechanics, staging, and imaging findings. Radiographics 2019;39:1437-60.

21. Leonard MH. Injuries of the lateral ligaments of the ankle; a clinical and experimental study. J Bone Joint Surg Am 1949;31A:373-7.

22. Kamada K, Watanabe S, Yamamoto H. Chronic subtalar instability due to insufficiency of the calcaneofibular ligament: a case report. Foot ankle Int 2002;23:1135-7.

23. Tochigi Y, Amendola A, Rudert MJ, Baer TE, Brown TD, Hillis SL, Saltzman CL. The Role of the Interosseous 
Talocalcaneal Ligament in Subtalar Joint Stability. Foot Ankle Int 2004;25:588-96.

24. Beltran J, Noto AM, Mosure JC, Shamam OM, Weiss KL, Zuelzer WA. Ankle: surface coil MR imaging at $1.5 \mathrm{~T}$. Radiology 1986;161:203-9.

25. Vandenbroucke JP, von Elm E, Altman DG, Gøtzsche PC, Mulrow CD, Pocock SJ, Poole C, Schlesselman JJ, Egger M; STROBE Initiative. Strengthening the Reporting of Observational Studies in Epidemiology (STROBE): explanation and elaboration. Int J Surg 2014;12:1500-24.

26. World Medical Association Declaration of Helsinki. Ethical principles for medical research involving human subjects. J Am Coll Dent 2014;81:14-8.

27. Mengiardi B, Pfirrmann CW, Vienne P, Kundert HP, Rippstein PF, Zollinger H, Hodler J, Zanetti M. Anterior Tibial Tendon Abnormalities: MR Imaging Findings. Radiology 2005;235:977-84.

28. Morrison WB, Carrino JA, Schweitzer ME, Sanders TG, Raiken DP, Johnson CE. Subtendinous bone marrow edema patterns on MR images of the ankle: association with symptoms and tendinopathy. AJR Am J Roentgenol 2001;176:1149-54.

29. Rosenberg ZS, Beltran J, Bencardino JT. From the RSNA Refresher Courses. Radiological Society of North America. MR imaging of the ankle and foot. Radiographics 2000;20 Spec No:S153-79.

30. Cheung Y, Rosenberg ZS, Magee T, Chinitz L. Normal anatomy and pathologic conditions of ankle tendons: current imaging techniques. RadioGraphics 1992;12:429-44.

31. Noto AM, Cheung Y, Rosenberg ZS, Norman A, Leeds NE. MR imaging of the ankle: normal variants. Radiology 1989;170:121-4.

32. Schweitzer ME, van Leersum M, Ehrlich SS, Wapner K. Fluid in normal and abnormal ankle joints: amount and distribution as seen on MR images. AJR Am J Roentgenol 1994;162:111-4.

33. Link SC, Erickson SJ, Timins ME. MR imaging of the ankle and foot: normal structures and anatomic variants that may simulate disease. AJR Am J Roentgenol 1993;161:607-12.

34. Berkowitz JF, Kier R, Rudicel S. Plantar fasciitis: MR imaging. Radiology 1991;179:665-7.

35. König H, Sieper J, Wolf KJ. Rheumatoid arthritis: evaluation of hypervascular and fibrous pannus with dynamic MR imaging enhanced with Gd-DTPA. Radiology 1990;176:473-7.

36. Bencardino J, Rosenberg ZS. MR Imaging of the Ankle and Hindfoot. Curr Protoc Magn Reson Imaging 2008;15:A25.1.1-A25.1.12.
37. Cohen J. A power primer. Psychol Bull 1992;112:155-9.

38. Debieux P, Wajnsztejn A, Mansur NSB. Epidemiology of injuries due to ankle sprain diagnosed in an orthopedic emergency room. Einstein (São Paulo) 2019;18:eAO4739.

39. Sijbrandij ES, van Gils APG, Louwerens JWK, de Lange EE. Posttraumatic Subchondral Bone Contusions and Fractures of the Talotibial Joint. AJR Am J Roentgenol 2000;175:1707-10.

40. Noback PC, Freibott CE, Tantigate D, Jang E, Greisberg JK, Wong T, Vosseller JT. Prevalence of Asymptomatic Achilles Tendinosis. Foot Ankle Int 2018;39:1205-9.

41. Zellers JA, Bley BC, Pohlig RT, Alghamdi NH, Silbernagel KG. Frequency of pathology on diagnostic ultrasound and relationship to patient demographics in individuals with insertional Achilles tendinopathy. Int J Sports Phys Ther 2019;14:761-9.

42. Dreyer MA, Dookie A. A Case of Neglected Achilles Rupture after an Ankle Sprain. Mil Med 2019;184:e306-e310.

43. Lam KW, Lui TH. Unrecognised Acute Rupture of the Achilles Tendon in Severe Ankle Sprain. J Orthop Trauma Rehabil 2015;19:97-9.

44. Ziai P, Benca E, von Skrbensky G, Graf A, Wenzel F, Basad E, Windhager R, Buchhorn T. The role of the peroneal tendons in passive stabilisation of the ankle joint: an in vitro study. Knee Surg Sports Traumatol Arthrosc 2013;21:1404-8.

45. Abdeen R, Comfort P, Starbuck C, Nester C. Ultrasound Characteristics of Foot and Ankle Structures in Healthy, Coper, and Chronically Unstable Ankles. J Ultrasound Med 2019;38:917-26.

46. Lee KM, Chung CY, Kwon SS, Chung MK, Won SH, Lee SY, Park MS. Relationship between stress ankle radiographs and injured ligaments on MRI. Skeletal Radiol 2013;42:1537-42.

47. Hembree WC, Wittstein JR, Vinson EN, Queen RM, Larose CR, Singh K, Easley ME. Magnetic Resonance Imaging Features of Osteochondral Lesions of the Talus. Foot Ankle Int 2012;33:591-7.

Cite this article as: Casado-Hernández I, Becerro-de-BengoaVallejo R, Losa-Iglesias ME, Santiago-Nuño F, MazoterasPardo V, López-López D, Rodríguez-Sanz D, Calvo-Lobo C. Association between anterior talofibular ligament injury and ankle tendon, ligament, and joint conditions revealed by magnetic resonance imaging. Quant Imaging Med Surg 2021;11(1):84-94. doi: 10.21037/qims-20-5 